\title{
Maritime mammals: terrestrial mammals as consumers in marine intertidal communities
}

\author{
James T. Carlton ${ }^{1, *}$, Janet Hodder ${ }^{2}$ \\ ${ }^{1}$ Maritime Studies Program, Williams College - Mystic Seaport, PO Box 6000, Mystic, Connecticut 06355, USA \\ ${ }^{2}$ Oregon Institute of Marine Biology, University of Oregon, PO Box 5389, Charleston, Oregon 97420, USA
}

\begin{abstract}
The phenomenon of terrestrial mammals as predators in marine intertidal communities, and thus as agents of energy transfer from sea to land, is poorly understood. We review here the evidence for terrestrial mammals intentionally entering the ocean shore at low tide in order to prey on living marine invertebrates, fish, algae, and seagrasses. We introduce the term 'maritime mammals', defined as coastal mammalian predators that utilize living intertidal energy resources and transfer these resources to the land. We document 135 records of predation among 45 species of terrestrial mammals in 8 orders feeding in marine intertidal zones. Most predation events are by carnivores (59\%, mostly by raccoon, mink, black bear, and Arctic fox), followed by rodents (20\%) and artiodactyls (14\%). Maritime mammals occur on all continental coastlines of the world except Antarctica. Most records are from the Eastern North Pacific Ocean, with 21 species of maritime mammals. Twelve maritime mammal species occur in the Western North Atlantic Ocean, and 8 maritime mammals are known for the Eastern South Pacific Ocean. These 3 regions account for $42 \%$ of the diversity of maritime mammals. A total of 228 different prey taxa are known to be consumed, representing 12 phyla of marine organisms; $2 / 3$ of these taxa are bivalve and gastropod mollusks, crabs, and fish. Introduced populations of 17 species of mammals have been recorded as maritime predators; 15 of these are recorded on islands where endemic mammals were absent or rare. Maritime mammals are widespread globally, with often repeated cases of predation being observed for the same species. We suggest that predation by maritime mammals is a rarely studied, rather than rare, phenomenon, and maritime mammals are thus a largely overlooked guild of intertidal predators. Quantitative field observations (using techniques such as infrared night vision, radiotelemetry, and stable isotope analysis) and experimental studies (using exclosures, or by taking advantage of the removal of insular introduced mammal populations) are required. The importance of intertidal resources in supplying energy to terrestrial populations of many species of mammals world wide may have been underappreciated.
\end{abstract}

KEY WORDS: Intertidal predators · Intertidal prey · Invasions · Mammal predation · Intertidal communities $\cdot$ Subsidies

\section{INTRODUCTION}

The amount of energy transferred between trophic levels, and the mechanisms by which that energy flows, are major organizing phenomena and processes of community structure (Krebs 1993, Ricklefs \& Miller 1999). In addition, the transfer of energy between ecosystems is widely recognized as an important component of food webs (Polis et al. 1997, Stapp et al.
1999). Energy-flow pathways between marine ecosystems include a variety of phenomena. A prominent example is the transport of detritus from one ecosystem to another. For example, drift algae and carcasses of marine vertebrates are commonly washed ashore and utilized by terrestrial consumers (Polis \& Hurd 1996a,b). Coastal algae and seagrasses are exported to the open ocean and the deep sea (Wolff 1976, Zieman et al. 1979, Graham et al. 2003). An equally common 
phenomenon is the utilization of prey in one habitat by predators from another habitat: terrestrial carnivores prey upon sea turtle eggs buried in sandy beaches (Hirth 1987, National Research Council 1990), terrestrial herbivores feed on intertidal salt marsh vegetation (Shanholtzer 1974), and deep sea plankton and nekton vertically migrate to prey upon organisms in upper ocean strata (Vinogradov 1968).

Energy derived from such allochthonous detritus or prey can subsequently flow into yet other parts of an adjacent ecosystem. Examples include bear feces composed of salmon contributing to energy flow in forests (Willson et al. 1998), and seabird feces composed of fish contributing to energy flow in other terrestrial biomes (Heatwole 1971, Crawford \& Shelton 1978). Other major between-ecosystem energy transfers involve long-distance migration of some species of insects, fish, birds, mammals, and other organisms. All of these cross-ecosystem energy flows may have important influences on the abundance, distribution and interactions of prey, predator, and detritivore populations, and thus the structuring of communities.

Within this context, the phenomenon of strictly terrestrial mammals as consumers, and thus as agents of energy transfer, in marine intertidal communities is poorly understood (Ricketts et al. 1952, Navarrete \& Castilla 1993). While the flow of energy from the sea to the land has come under increased scrutiny (Pollis \& Hurd 1996a,b, Stapp et al. 1999), the majority of reviews of marine littoral ecosystems make little or no reference to terrestrial mammals as intertidal predators (Valiela 1995, Raffaelli \& Hawkins 1996, Nybakken 2000, Levinton 2001), and few direct studies exist that focus on this phenomenon (Navarrete \& Castilla 1993, Conradt 2000).

This lack of recognition (and thus understanding) of the phenomenon of terrestrial mammals as intertidal consumers may arise from at least 4 situations: (1) The phenomenon may be naturally rare: predation on marine organisms by terrestrial mammals may be rare both in time and space, and thus not a significant mechanism of either interecosystem energy flow or of the structuring of intertidal communities; (2) The phenomenon may be rarer now: in some regions, because of the extirpation by humans of both predator and prey from urbanized coastal zones, once morecommon predation events may now be infrequent or no longer occur. Indeed, most coastal observation stations (such as marine biological laboratories) are now in relatively urbanized areas. An example may be the observation of predation by bears on intertidal lobsters in Maine. John Josselyn, writing in 1674 of observations made in 1638 and in 1663, wrote that 'The bear is a tyrant at a lobster and at low water will go down to the rocks after them with great diligence', an observation apparently not made since the 17 th century (Table 1). In some instances however, predation by maritime mammals may be enhanced by human activities. Urbanization may lead to higher densities or new populations of native or introduced predators. High densities of domestic animals close to the shore may also lead to increased interactions with the marine environment; (3) The phenomenon may be highly localized: predation on marine organisms by terrestrial mammals may occur only in certain localized regions, albeit being common at such sites (Navarrete \& Castilla 1993); (4) The phenomenon may be relatively common but unrecognized and underreported: predation on marine organisms by terrestrial mammals may be common but largely underappreciated or underdescribed, for at least 5 reasons. These are, (i) it is rarely reported in easily accessible literature, (ii) incidental observations by marine ecologists and others are over time increasingly less likely to be published because of their nonquantitative or nonexperimental nature, (iii) it may be underrecorded, being a primarily crepuscular or nocturnal low tide activity (Moore 2002), (iv) it falls at the interface of the disciplines of marine and terrestrial ecology and is thus rarely studied (Navarette \& Castilla 1993), or (v) experimental studies on the importance of terrestrial mammals as intertidal predators are non-existent, and thus it has been presumed to be insignificant both in terms of an energy flow pathway and as an organizing force in intertidal community structure.

We review here (and provide previously unpublished data on) the phenomenon of terrestrial mammal predation in coastal ecosystems worldwide. Our focus is on the evidence for terrestrial mammals intentionally entering the ocean shore at low tide in order to consume living (not detrital) marine invertebrates, fish, algae, and seagrasses. We call this consumption predation. We introduce the term 'maritime mammals', defined as coastal mammalian predators that utilize living intertidal energy resources and transfer these resources to the land. We exclude from our review 3 phenomena which also bring terrestrial mammals into maritime arenas: (1) predation on seabird and sea turtle eggs and hatchlings; (2) the scavenging of carrion and detritus on beaches; and (3) predation in salt marshes and mangroves. These 3 aspects are reviewed in part by Moore (2002). We have excluded in particular those reports of domestic animals (such as cows, horses, and pigs) feeding on intertidal algae where it is unclear if these herbivores are feeding on attached living seaweed or on drift plant material. This noted, the consumption of seaweed wrack is common enough in parts of Europe such that certain algae have been given common names such as pig weed, cow weed, and horse weed 
(Moore 2002). We also exclude the activities of human (Homo sapiens) predators and foragers in this review.

We further examine these data for evidence of the relative roles of introduced (non-indigenous) versus native predators, given the predominance of certain species of introduced mammals on islands and in coastal zones (King 1985). We also consider the role of introduced prey species in maritime mammal diets. We further consider hypotheses that address why terrestrial mammals would seek food resources in the intertidal zone, and the spatial or temporal circumstances under which such predation may occur. We review biases in the nature and quality of the data, and then ask whether the phenomenon of maritime mammals is rare, highly localized, or perhaps common but largely unrecognized.

Finally, we discuss the evidence for, and research needs to determine, the potential ecological importance of this phenomenon relative to both landward energy flow and to altering the diversity, abundance and distribution of intertidal prey species.

\section{MATERIALS AND METHODS}

We searched the literature for reports of predation by terrestrial mammals on marine intertidal animals and plants and interviewed colleagues familiar with intertidal shores. In some instances we admit reliable reports of observations of mammals foraging and consuming organisms in the intertidal zone, although the exact nature of the prey is not yet known. Most published observations occur as incidental remarks, and we thus assume that we have missed reports. We report our own field observations derived from western and eastern North America (Oregon, California, and Connecticut).

For analysis of prey diversity, we scored individual species where reported. For prey reported only at higher taxonomic levels (e.g. brachyurans, crustaceans, tide pool fish, bivalves, and so forth) we conservatively scored these as unique taxa only if the same type of organism in the same geographic region was not already reported at the species level for that predator. For example, where the brachyuran crab Cancer is reported as prey of mink in Washington, and brachyurans as prey of mink in nearby British Columbia, we scored these reported prey as only one species for both regions. However, if distinctly different geographic regions were involved, we scored unidentified prey as different species. For example, where the brachyuran crab Ocypode is reported as prey of domestic dogs in Fiji, and brachyura as prey of dogs in Puerto Rico, we scored these prey as 2 different species.

\section{RESULTS}

\section{Biogeography and diversity of maritime mammals}

We document 135 records of predation among 45 species of terrestrial mammals in 8 orders (Tables 1 \& 2) feeding in marine intertidal zones. Eighty-seven percent of the mammalian predators are in 3 orders (Carnivora 44\%, Rodentia 27\%, and Artiodactyla $16 \%$ ). The remaining orders (marsupials [Didelphimorphia and Dasyuromorphia], Primates, Lagomorpha, and Insectivora) are represented by far fewer species. Most predation events are by Carnivora (59\%, mostly by raccoon, mink, black bear, and Arctic fox), followed by Rodentia $(20 \%$, over $1 / 3$ of which are the Norway rat), and Artiodactyla (14\%, over half of which are domestic pigs and mule deer).

No living terrestrial mammal species are known to specialize entirely on intertidal prey. Sheep, however, on North Ronaldsay and Linga-Holme on the Orkney Islands in Scotland are known to live almost exclusively on intertidal seaweeds (Hall 1975, Conradt 2000). We note the existence of the poorly known and now-extinct sea mink Mustela macrodon, last verifiably seen in 1880 (Carlton et al. 1999). This mink lived on the coastal zone from Canada to Connecticut, and was hunted to extinction for its fur. It appears to have been restricted to rocky shore habitats both on the mainland and on offshore islands, and may have spent most of its time in the water (Manville 1966, Campbell 1988). It may have preyed almost entirely on marine intertidal and shallow sublittoral prey, although the only verified prey were intertidal fish (Manville 1966). If the sea mink was a specialized maritime mammal, then the only such species has become extinct.

\section{Biogeographic patterns}

Maritime mammals occur on all continental coastlines of the world except Antarctica. Most records are from the Eastern North Pacific Ocean (Alaska to the Galapagos Islands) with 21 species of maritime mammals known, of which 12 are unique (but not necessarily endemic) to this region (Beechey ground squirrel, deer mouse, Keen's mouse, muskrat, Townsend's vole, coyote, gray fox, red fox, grizzly bear, American pine marten, mule deer, mountain goat).

Twelve maritime mammal species are reported for the Western North Atlantic Ocean (Greenland to the Caribbean, including the Gulf of Mexico), of which 3 are unique records (opossum, masked shrew, and gray wolf). Eight maritime mammals are known for the Eastern South Pacific Ocean (the west coast of South 
Table 1. Terrestrial mammals feeding in the intertidal zone. Common and Latin mammal names follow Nowak (1999). Prey that are introduced in the localities are indicated by Latin names in bold. Abbreviations are as follows: Summary of prey taxa ( $\left.\sum \mathrm{P}\right)$ : $\mathrm{A}=$ no. of different animal species consumed, $\mathrm{P}=$ no. of different plant species consumed; native/introduced status of mammals: $\mathrm{N}=$ native, $\mathrm{I}=$ introduced; observations (if not shown, nature of observation is not given by author): $\mathrm{B}=$ material in burrows or dens, $\mathrm{DO}=$ direct observation of feeding, $\mathrm{S}=$ scat (feces), $\mathrm{SI}=$ based on stable isotopes, $\mathrm{ST}=$ stomach or digestive tract contents

\begin{tabular}{|c|c|c|c|c|}
\hline Order/Species/ $/ \mathrm{P}$ & N/I & Location/(observations) & Prey & Source \\
\hline \multicolumn{5}{|l|}{ Didelphimorphia } \\
\hline \multirow{2}{*}{$\begin{array}{l}\text { Virginia opossum } \\
\text { (Didelphis virginiana) A:1 }\end{array}$} & $\mathrm{N}$ & USA: Gulf of Mexico & Brachyura (Uca pugilator) & Rathbun (1918) \\
\hline & $\mathrm{N}$ & USA: Gulf of Mexico & $\begin{array}{l}\text { 'Living among jetty rocks and feeding } \\
\text { upon the sessile mid-littoral jetty fauna' }\end{array}$ & Britton \& Morton (1989) \\
\hline $\begin{array}{l}\text { Dasyuromorphia } \\
\text { Tasmanian devil } \\
\text { (Sarcophilus harrisii) A:1 }\end{array}$ & $\mathrm{N}$ & Australia: Tasmania & Amphipoda (Talitridae) & Moore (2002) \\
\hline $\begin{array}{l}\text { Insectivora } \\
\text { Lesser white-toothed shrew } \\
\text { (Crocidura suaveolens) A:1 }\end{array}$ & $\mathrm{N}$ & UK: Scilly Islands & $\begin{array}{l}\text { Amphipoda (probably Talitridae) } \\
\text { 'As well as other small invertebrates' }\end{array}$ & Churchfield (1990) \\
\hline $\begin{array}{l}\text { Masked shrew } \\
\text { (Sorex cinereus) A:2 }\end{array}$ & $\mathrm{N}$ & $\begin{array}{l}\text { Canada: Nova Scotia } \\
\text { Bon Portage Isl. (ST) }\end{array}$ & $\begin{array}{l}\text { Amphipoda ('Littoral', possibly Tali- } \\
\text { tridae); Diptera (kelp flies, Coelopidae) }\end{array}$ & Stewart et al. (1989) \\
\hline \multicolumn{5}{|l|}{ Primates } \\
\hline \multirow{2}{*}{$\begin{array}{l}\text { Chacma baboon } \\
\text { (Papio ursinus) A:7 }\end{array}$} & $\mathrm{N}$ & South Africa & 'Marine shellfish and crustaceans' & Avery \& Siegfried (1980) \\
\hline & $\begin{array}{l}\mathrm{N} \\
\mathrm{N}\end{array}$ & $\begin{array}{l}\text { South Africa } \\
\text { South Africa: } \\
\text { Cape of Good } \\
\text { Hope (DO, S) }\end{array}$ & $\begin{array}{l}\text { Amphipoda (Talitridae) } \\
\text { Bivalvia ('black mussels': Choromytilus } \\
\text { meridionalis); Gastropoda ('periwinkles', } \\
\text { 'limpets'); Amphipoda (Talorchestia } \\
\text { sp.); Isopoda ('rock lice', possibly Ligia); } \\
\text { Brachyura (Cyclograpsus punctatus; } \\
\text { Plagusia chabrus) }\end{array}$ & $\begin{array}{l}\text { Brown \& McLachlan (1990) } \\
\text { Hall (1962) }\end{array}$ \\
\hline \multicolumn{5}{|l|}{ Lagomorpha } \\
\hline $\begin{array}{l}\text { Domestic rabbit (Oryctolagus } \\
\text { cuniculus) P:1 }\end{array}$ & $S$ I & $\begin{array}{l}\text { South Africa: Schaapen } \\
\text { Isl., Saldanha Bay (DO) }\end{array}$ & Algae (Ulva) & $\begin{array}{l}\text { G. Branch (pers. comm.); } \\
\text { Moore }(2002)\end{array}$ \\
\hline \multicolumn{5}{|l|}{ Rodentia } \\
\hline \multirow[t]{2}{*}{$\begin{array}{l}\text { Beechey ground squirrel } \\
\text { (Spermophilus beecheyi) P:1 }\end{array}$} & $\mathrm{N}$ & $\begin{array}{l}\text { USA: California: Monterey } \\
\text { Bay: Pacific Grove (DO) }\end{array}$ & $\begin{array}{l}\text { Foraging in intertidal zone: } \\
\text { prey not known }\end{array}$ & J. T. Carlton (pers. obs.) \\
\hline & $\mathrm{N}$ & $\begin{array}{l}\text { USA: California: } \\
\text { Morro Bay (DO) }\end{array}$ & $\begin{array}{l}\text { Eelgrass } \\
\text { (Zostera marina) }\end{array}$ & Roest (1993) \\
\hline \multirow[t]{2}{*}{$\begin{array}{l}\text { Deer mouse (Peromyscus } \\
\text { maniculatus) A:3 }\end{array}$} & $\mathrm{N}$ & $\begin{array}{l}\text { USA: California: } \\
\text { Humboldt County (ST) }\end{array}$ & $\begin{array}{l}\text { Amphipoda (Talitridae, Megalorchestia?); } \\
\text { Diptera Coleoptera, (Staphylinidae) }\end{array}$ & Osborne \& Sheppe (1971) \\
\hline & $\mathrm{N}$ & Mexico: Baja California & 'Intertidal invertebrates' & Stapp (1999) \\
\hline $\begin{array}{l}\text { Keen's mouse } \\
\text { (Peromyscus keeni) }\end{array}$ & $\mathrm{N}$ & $\begin{array}{l}\text { Canada: British Columbia: } \\
\text { Triangle Isl. (SI) }\end{array}$ & 'Intertidal organisms' & Drever et al. (2000) \\
\hline $\begin{array}{l}\text { Andean rat (Chroeomys } \\
\text { olivaceus) A:3, P:1 }\end{array}$ & $\mathrm{N}$ & Chile: Punta Metri (ST) & $\begin{array}{l}\text { Bivalvia (Mytilidae); Gastropoda; } \\
\text { Anomura (Petrolisthes sp.); algae }\end{array}$ & Martinez et al. (1986) \\
\hline $\begin{array}{l}\text { House mouse } \\
\text { (Mus musculus) A:2, P:1 }\end{array}$ & I & Chile: Las Cruces (ST) & $\begin{array}{l}\text { Gastropoda; Brachyura; } \\
\text { Rhodophyta }\end{array}$ & Navarrete \& Castilla (1993) \\
\hline $\begin{array}{l}\text { Mouse (unidentified) } \\
\text { A:1 }\end{array}$ & $\mathrm{N}$ ?/I? & $\begin{array}{l}\text { USA: Oregon: } \\
\text { Coos Bay (DO) }\end{array}$ & $\begin{array}{l}\text { Gastropoda } \\
\text { (limpet, Lottia digitalis) }\end{array}$ & Frank (1965) \\
\hline $\begin{array}{l}\text { Towsend's vole } \\
\text { (Microtus townsendii cowani) }\end{array}$ & i) & $\begin{array}{l}\text { Canada: British Columbia: } \\
\text { Triangle Isl. (SI) }\end{array}$ & 'Intertidal organisms' & Drever et al. (2000) \\
\hline $\begin{array}{l}\text { Muskrat (Ondatra zibethicus) } \\
\text { A:1 }\end{array}$ & N & $\begin{array}{l}\text { USA: Washington: } \\
\text { Puget Sound }\end{array}$ & $\begin{array}{l}\text { Bivalvia } \\
\text { (mussels, Mytilus) }\end{array}$ & Martinez et al. (1986) \\
\hline $\begin{array}{l}\text { False water rat (Xeromys } \\
\text { myoides) A:1 }\end{array}$ & $\mathrm{N}$ & $\begin{array}{l}\text { Australia: Victoria: } \\
\text { Queenscliff, Swan Bay (DO) }\end{array}$ & Brachyura (Carcinus maenas) & G. Parry (pers. comm.) \\
\hline \multirow{3}{*}{$\begin{array}{l}\text { House (black) rat (Rattus } \\
\text { rattus) A:16 }\end{array}$} & I & Midway Atoll (DO) & Brachyura (Uca sp.) & N. Seto (pers. comm.) \\
\hline & I & Chile: Punta Metri (ST) & Bivalvia (Mytilidae); Gastropoda & Martinez et al. (1986) \\
\hline & I & $\begin{array}{l}\text { Chile: Punta Kilian: } \\
\text { Mehuin (S) }\end{array}$ & $\begin{array}{l}\text { Bivalvia (Perumytilus purpuratus); } \\
\text { Gastropoda (Concholepas concholepas, } \\
\text { Fissurella picta, F. nigra, Siphonaria } \\
\text { lessoni, Scurria parasitica); Polyplaco- } \\
\text { phora (Chiton latus, C. granosus); Bra- } \\
\text { chyura (Acanthocyclus gayi, A. hassleri, }\end{array}$ & Zamorano (1986) \\
\hline
\end{tabular}


Table 1 (continued)

\begin{tabular}{|c|c|c|c|c|}
\hline Order/Species/ $/ \mathrm{P}$ & N/I & Location/(observations) & Prey & Source \\
\hline & & & $\begin{array}{l}\text { Pisoides edwardsi, Homalaspis plana); (Petro- } \\
\text { listhes violaceus); Anomura Fish (Sicyases } \\
\text { sanguineus, Calliclinus geniguttatus) }\end{array}$ & \\
\hline Rat (Norway? Black?) & $\mathrm{N}$ & Europe: Denmark? & Gastropoda (limpet Patella) & Thorson (1971) \\
\hline \multirow[t]{11}{*}{$\begin{array}{l}\text { Norway rat } \\
\text { (Rattus norvegicus) } \\
\text { A:66, P:1 }\end{array}$} & I & Italy: Po River valley & $\begin{array}{l}\text { Bivalvia (Cerastoderma lamarcki, Ensis sp., } \\
\text { Mactra corallina, Mytilus galloprovincialis, } \\
\text { Scrobicularia plana); Gastropoda (Haminoea } \\
\text { sp.); Brachyura (Carcinus aestuarii) }\end{array}$ & Parisi \& Gandolfi (1974) \\
\hline & I & UK: Isle of Man (D) & $\begin{array}{l}\text { 'Occasionally seen } \\
\text { foraging on the shore' }\end{array}$ & Thompson et al. (2000) \\
\hline & I & $\begin{array}{l}\text { USA: } \\
\text { Massachusetts }\end{array}$ & $\begin{array}{l}\text { Bivalvia; Gastropoda } \\
\text { (including Nucella lamellosa) }\end{array}$ & Menge \& Sutherland (1987) \\
\hline & I & USA: Atlantic coast & $\begin{array}{l}\text { 'Forage at low tide on invertebrates'; } \\
\text { (Brachyura, Ocypodidae) 'ghost crabs' }\end{array}$ & Amos (1966) \\
\hline & I & USA: Pacific coast & Brachyura (Pachygrapsus crassipes) & Garth \& Abbott (1980) \\
\hline & I & $\begin{array}{l}\text { Canada: Queen Charlotte } \\
\text { Islands }\end{array}$ & $\begin{array}{l}\text { Bivalvia; Gastropoda; Crustacea: } \\
\text { Amphipoda, Decapoda; Fish }\end{array}$ & Drever \& Harestad (1998) \\
\hline & I & $\begin{array}{l}\text { Chile: Las Cruces } \\
(\mathrm{B}, \mathrm{S})\end{array}$ & $\begin{array}{l}\text { Bivalvia (Perumytilus purpuratus, Brachidontes } \\
\text { granulata, Mesodesma donacium, Tagelus } \\
\text { dombeii); (Gastropoda Fissurella crassa, } \\
\text { F. axima, F. Costata, F. limbata, Collisella spp., } \\
\text { Scurria scurra, S. parasitica, Prisogaster niger, } \\
\text { Tegula atra, Diloma nigerrima, Littorina peru- } \\
\text { viana, Siphonaria lessoni, Concholepas concho- } \\
\text { lepas, Trimusculus peruvianus); Polyplacophora } \\
\text { (Chiton granosus, C. spp.); Cirripedia (Austro- } \\
\text { megabalanus psittacus); Brachyura (Allopetro- } \\
\text { isthes punctatus, Leptograpsus variegatus, } \\
\text { Acanthocyclus gayi, A. hassleri, Paraxanthus } \\
\text { barbiger; Homalaspis plana, Taliepus dentatus); } \\
\text { Anomura (Petrolisthes violaceus, P. tuberculatus } \\
\text { P. granulosus, P. laevigatus, Pachycheles grossi- } \\
\text { manus); Asteroidea (Heliaster helianthus); Echi- } \\
\text { noidea (Loxechinus albus); Fish (Aphos porosus, } \\
\text { Sicyases sanguineus) }\end{array}$ & Navarrete \& Castilla (1993) \\
\hline & I & Chile: Punta Metri (ST) & $\begin{array}{l}\text { Bivalvia (Mytilidae); Gastropoda; } \\
\text { Anomura (Petrolisthes sp.); algae }\end{array}$ & Martinez et al. (1986) \\
\hline & I & Chile: Las Cruces (ST) & $\begin{array}{l}\text { Polychaeta; Gastropoda; } \\
\text { Brachyura; Rhodophyta }\end{array}$ & Navarrete \& Castilla (1993) \\
\hline & I & $\begin{array}{l}\text { New Zealand: } \\
\text { Noises Isl. (S, ST) }\end{array}$ & $\begin{array}{l}\text { Polychaeta Bivalvia (mussels, Xenostrobus } \\
\text { pulex); Gastropoda (Onchidella sp., cf. O. } \\
\text { nigricans; Cellana spp., Nerita atramentosa; } \\
\text { Eatoniella sp. Lepsiella scobina, Diloma sp.); } \\
\text { Cirripedia (Chamaesipho columna); Brachyura } \\
\text { (Pinnotheres novaezelandiae); }\end{array}$ & Moors (1985) \\
\hline & I & New Zealand: Ike Isl. (B) & $\begin{array}{l}\text { Gastropoda (Turbo smaragdus, Thais } \\
\text { orbita); Brachyura (Leptograpsus variegatus); }\end{array}$ & Moors (1985) \\
\hline $\begin{array}{l}\text { Polynesian rat } \\
\text { (Rattus exulans) A:1 }\end{array}$ & I & $\begin{array}{l}\text { Midway Atoll } \\
\text { (DO) }\end{array}$ & $\begin{array}{l}\text { Brachyura } \\
\text { (Uca sp.) }\end{array}$ & N. Seto (pers. comm.) \\
\hline $\begin{array}{l}\text { Pygmy rice rat (Oligo- } \\
\text { ryzomys longicau- } \\
\text { datus) A:1, P:1 }\end{array}$ & $\mathrm{N}$ & Chile: Punta Metri (ST) & Gastropoda; algae & Martinez et al. (1986) \\
\hline $\begin{array}{l}\text { Carnivora } \\
\text { Raccoon dog (Nycte- } \\
\text { reutes procyonoides }) \\
\text { A:2 }\end{array}$ & $\mathrm{N}$ & Russia: Pacific coast & Brachyura; Echinoidea & Novikov (1956) \\
\hline \multirow[t]{2}{*}{$\begin{array}{l}\text { Coyote (Canis latrans) } \\
\mathrm{A}: 7, \mathrm{P}: 2\end{array}$} & $\mathrm{~N}$ & $\begin{array}{l}\text { Mexico: Baja California } \\
\text { Santa Maria Bay (DO) }\end{array}$ & Bivalvia ('clams') fish ('puffer fish') & Crawford (1953) \\
\hline & $\mathrm{N}$ & $\begin{array}{l}\text { Mexico: Baja California: } \\
\text { Gulf of California (S) }\end{array}$ & $\begin{array}{l}\text { Polychaeta ('tube worms'); Gastropoda } \\
\text { ('snails'); Isopoda (Ligia, Tylos); Brachyura } \\
\text { (Ocypodidae); Chlorophyta; Phaeophyta }\end{array}$ & Rose \& Polis (1998) \\
\hline
\end{tabular}


Table 1 (continued)

\begin{tabular}{|c|c|c|c|c|}
\hline Order/Species/¿P & N/I & Location/(observations) & Prey & Source \\
\hline & $\mathrm{N}$ & Mexico: Baja California & 'A consistent intertidal feeder' & Ricketts et al. (1952) \\
\hline Gray wolf A:1 (Canis lupus) & $\mathrm{N}$ & USA: Gulf of Mexico & Brachyura (Uca pugilator) & Rathbun (1918) \\
\hline \multirow{5}{*}{$\begin{array}{l}\text { Domestic dog } \\
\text { (Canis famliaris) A:5 }\end{array}$} & I & Puerto Rico & Brachyura ('crabs') & Crosby (1972) \\
\hline & I & $\begin{array}{l}\text { USA: Maine: } \\
\text { Vineyard Haven (DO) }\end{array}$ & Cirripedia (Semibalanus balanoides) & J. T. Carlton (pers. obs.) \\
\hline & I & Fiji: Yanuca Isl. (DO) & Brachyura (Ocypodidae, Ocypode sp.) & $\begin{array}{l}\text { L. Eldredge \& } \\
\text { J. T. Carlton (pers. obs.) }\end{array}$ \\
\hline & I & $\begin{array}{l}\text { Kiribati: Canton } \\
\text { Atoll (DO) }\end{array}$ & Fish (mullet) & $\begin{array}{l}\text { Degener \& Gillaspy (1955) } \\
\text { P. Jokiel (pers. comm.) }\end{array}$ \\
\hline & I & Peru: Atacama coast & 'shellfish' & Crosby (1972) \\
\hline \multirow{2}{*}{$\begin{array}{l}\text { Black-backed jackal } \\
\text { (Canis mesomelas) A:2 }\end{array}$} & $\mathrm{N}$ & Namibia (DO) & Fish (Mugil cephalus) & Nel \& Loutit (1986) \\
\hline & $\mathrm{N}$ & $\begin{array}{l}\text { Namibia: Cape } \\
\text { Cross Seal Reserve (S) }\end{array}$ & Bivalvia ('brown mussel') & Hiscocks \& Perrin (1987) \\
\hline \multirow[t]{10}{*}{$\begin{array}{l}\text { Arctic fox (Alopex } \\
\text { lagopus) A:29, P: } 2\end{array}$} & $\mathrm{~N}$ & Iceland (S) & $\begin{array}{l}\text { Mollusca; Crustacea; Insecta (Diptera, } \\
\text { 'kelp flies'); algae ('seaweed') }\end{array}$ & $\begin{array}{l}\text { Hersteinsson \& MacDonald } \\
\text { (1996) }\end{array}$ \\
\hline & $\mathrm{N}$ & Iceland & Fish (Cyclopterus lumpus) & Braestrup (1941) \\
\hline & $\mathrm{N}$ & Greenland & $\begin{array}{l}\text { Porifera; Polychaeta ('worms'); Bivalvia } \\
\text { (Mytilus edulis, Mya arenaria, M. tyrun- } \\
\text { cata); Gastropda (including 'sea slugs'); } \\
\text { Brachyura (Hyas areneus, Chionoecetes } \\
\text { opilio) Asteroidea; Echinoidea; Holo- } \\
\text { thuroidea; Ascidiacea; Fish (Pholis gunellu, } \\
\text { Lumpenus fabricus Mallotus villosus, } \\
\text { Gadus saidua) }\end{array}$ & Braestrup (1941) \\
\hline & $\mathrm{N}$ & Greenland (DO) & $\begin{array}{l}\text { Fish (Pholis fasciatus, Cyclopterus } \\
\text { lumpus, Myoxocepalus sp. 'and others') }\end{array}$ & Nielsen (1991) \\
\hline & $\mathrm{N}$ & Greenland (ST) & 'Fish, shellfish and seaweed' & Kapel (1999) \\
\hline & $\mathrm{N}$ & Russia: Arctic island & $\begin{array}{l}\text { 'Marine invertebrates important in } \\
\text { summer diet' }\end{array}$ & Lavrov (1932), Fox (1975) \\
\hline & $\mathrm{N}$ & $\begin{array}{l}\text { Russia: Commander } \\
\text { Islands }\end{array}$ & $\begin{array}{l}\text { Porifera; Polychaeta ('worms'); Mollusca; } \\
\text { 'crustaceans'; Echinoidea; Holothuroidea; } \\
\text { Ascidiacea }\end{array}$ & Novikov (1956) \\
\hline & I & $\begin{array}{l}\text { USA: Alaska: } \\
\text { Aleutian Islands } \\
\text { Nizki/Alaid Islands (ST) }\end{array}$ & $\begin{array}{l}\text { Porifera; Cirripedia; Amphipoda; } \\
\text { Brachyura; Holothuroidea; } \\
\text { Phaeophyta (kelp) }\end{array}$ & West (1987) \\
\hline & I & $\begin{array}{l}\text { USA: Alaska: } \\
\text { Aleutian Islands: } \\
\text { Kagalaska Isl. (S) }\end{array}$ & $\begin{array}{l}\text { Bivalvia (mussels,clams); Gastropoda; } \\
\text { Cirripedia; Amphipoda; Isopoda; } \\
\text { Brachyura; Echinoidea; Holothuroidea; } \\
\text { Phaeophyta (kelp) }\end{array}$ & West (1987) \\
\hline & I & $\begin{array}{l}\text { USA: Alaska: } \\
\text { Aleutian Islands (S) }\end{array}$ & $\begin{array}{l}\text { Polychaeta (Nereis); Bivalvia (clams, } \\
\text { mussels); Gastropoda; Amphipoda; } \\
\text { Cirripedia (Pollicipes polymerus); } \\
\text { Isopoda; Echinoidea }\end{array}$ & Murie (1959) \\
\hline $\begin{array}{l}\text { Gray fox (Urocyon } \\
\text { cinereoargenteus) A: } 3\end{array}$ & $\mathrm{~N}$ & $\begin{array}{l}\text { Mexico: Baja California } \\
\text { Gulf of California (DO) }\end{array}$ & $\begin{array}{l}\text { Gastropoda; Brachyura } \\
\left({ }^{\prime} \text { crabs'); Holothuroidea }^{2}\right.\end{array}$ & Rose \& Polis (1998) \\
\hline $\begin{array}{l}\text { Patagonian fox (Pseu- } \\
\text { dalopex griseus) A:3 }\end{array}$ & I & Falkland Islands & $\begin{array}{l}\text { Gastropoda (limpets); Brachyura ('crabs'); } \\
\text { fish (dogfish, Harpagifer hispinis) }\end{array}$ & Strange (1983) \\
\hline $\begin{array}{l}\text { Peruvian desert fox } \\
\text { (Dusicyon sechurae) A:1 }\end{array}$ & $\mathrm{N}$ & $\begin{array}{l}\text { Peru: ocean } \\
\text { beaches adjacent } \\
\text { to Sechura Desert (S) }\end{array}$ & Brachyura ('crabs') & Huey (1969) \\
\hline $\begin{array}{l}\text { Red fox } \\
\text { (Vulpes vulpes) }\end{array}$ & I & $\begin{array}{l}\text { USA: Washington: } \\
\text { San Juan Isl. (DO) }\end{array}$ & $\begin{array}{l}\text { Foraging in intertidal: } \\
\text { prey not known }\end{array}$ & $\begin{array}{l}\text { Holmes (1992), } \\
\text { M. Herko (pers. comm.) }\end{array}$ \\
\hline Red or gray fox & N/I & $\begin{array}{l}\text { Pacific American coast: } \\
\text { 'islands to the far north' }\end{array}$ & $\begin{array}{l}\text { Amphipoda ('Traskorchestia } \\
\text { and other amphipods') }\end{array}$ & Ricketts et al. (1986) \\
\hline $\begin{array}{l}\text { Brown hyena } \\
\text { (Parahyaena brunnea) A:1 }\end{array}$ & $\mathrm{N}$ & $\begin{array}{l}\text { Namibia: } \\
\text { Sandwich Harbor (S) }\end{array}$ & Brachyura & Stuart \& Shaughnessy(1984) \\
\hline $\begin{array}{l}\text { Striped hyena } \\
\text { (Hyaena hyaena) A:1 }\end{array}$ & $\mathrm{N}$ & Kenya & Brachyura (open coast sand beach species) & $\begin{array}{l}\text { National Geographic } \\
\text { Society (1998) }\end{array}$ \\
\hline
\end{tabular}


Table 1 (continued)

\begin{tabular}{|c|c|c|c|c|}
\hline 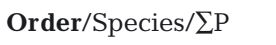 & $\mathrm{N} / \mathrm{I}$ & Location/(observations) & Prey & Source \\
\hline \multirow[t]{9}{*}{$\begin{array}{l}\text { American black bear } \\
\text { (Ursus americanus) } \\
\text { A:6, P:1 }\end{array}$} & $\mathrm{N}$ & $\begin{array}{l}\text { USA Maine Canada: } \\
\text { British Columbia: } \\
\text { Clayoquot Sound (DO) }\end{array}$ & $\begin{array}{l}\text { Astacura (lobster, Homarus americanus) } \\
\text { Cirripedia (Balanus glandula); Brachyura } \\
\text { ('shore crabs' [Grapsidae]); Asteroidea }\end{array}$ & $\begin{array}{l}\text { Josselyn (1674) } \\
\text { (in Conkling 1999) } \\
\text { Oldershaw (1995) }\end{array}$ \\
\hline & $\mathrm{N}$ & $\begin{array}{l}\text { Canada: Queen } \\
\text { Charlotte Islands (DO) }\end{array}$ & $\begin{array}{l}\text { Amphipoda (Talitridae, Traskorchestia } \\
\text { traskiana); Brachyura (Hemigrapsus nudus) }\end{array}$ & Ellis \& Wilson (1981) \\
\hline & $\mathrm{N}$ & $\begin{array}{l}\text { Canada: } \\
\text { Vancouver Isl.: Bamfield }\end{array}$ & $\begin{array}{l}\text { Rolling large cobbles and } \\
\text { eating barnacles (Cirripedia) }\end{array}$ & D. Padilla (pers. comm.) \\
\hline & $\mathrm{N}$ & $\begin{array}{l}\text { Canada: } \\
\text { Vancouver Isl. }\end{array}$ & $\begin{array}{l}\text { Photograph of black bear flipping rocks } \\
\text { in search of crabs (Brachyura) }\end{array}$ & Ramsay (1985) \\
\hline & $\mathrm{N}$ & $\begin{array}{l}\text { Canada: Queen Charlotte } \\
\text { Islands Princess Royal Isl. (DO) }\end{array}$ & $\begin{array}{l}\text { Scraping barnacles off } \\
\text { intertidal rocks }\end{array}$ & E. A. Mills (pers. comm.) \\
\hline & $\mathrm{N}$ & USA: Alaska: southern & Phaeophyta (Fucus gardneri) & O'Clair \& O'Clair (1998) \\
\hline & $\mathrm{N}$ & $\begin{array}{l}\text { USA: Alaska: Katmai } \\
\text { National Park Shelikof Strait }\end{array}$ & Bivalvia (mussel, Mytilus californianus) & T. Klinger (pers. comm.) \\
\hline & $\mathrm{N}$ & $\begin{array}{l}\text { USA: Alaska: } \\
\text { Prince William Sound }\end{array}$ & Foraging in mussel beds: prey not known & C. Hewitt (pers. comm.) \\
\hline & $\mathrm{N}$ & $\begin{array}{l}\text { USA: Alaska: Tracy } \\
\text { Arm (South of Juneau) and } \\
\text { Glacier Bay National Park } \\
(\mathrm{DO}, \mathrm{S})\end{array}$ & $\begin{array}{l}\text { Video of bears scraping barnacles off } \\
\text { intertidal rocks; scat consists of barnacle } \\
\text { remains }\end{array}$ & N. Folsom (pers. comm.) \\
\hline \multirow[t]{4}{*}{$\begin{array}{l}\text { Grizzly (brown) bear } \\
\text { (Ursus arctos) A:3 }\end{array}$} & $\mathrm{N}$ & $\begin{array}{l}\text { USA: Alaska: Kenai } \\
\text { Peninsula: Kachemak } \\
\text { Bay, Ishmaloff Isl. (DO) }\end{array}$ & $\begin{array}{l}\text { Bivalvia } \\
\text { (Saxidomus giganteus) }\end{array}$ & M. Matthews (pers. comm.) \\
\hline & $\mathrm{N}$ & $\begin{array}{l}\text { USA: Alaska: Katmai } \\
\text { National Park Shelikof } \\
\text { Strait (DO) }\end{array}$ & Bivalvia (Siliqua patula) & E. A. Mills (pers. comm.) \\
\hline & $\mathrm{N}$ & $\begin{array}{l}\text { USA: Alaska: Katmai } \\
\text { National Park Shelikof Strait }\end{array}$ & Foraging in intertidal: prey not known & T. Klinger (pers. comm.) \\
\hline & $\mathrm{N}$ & $\begin{array}{l}\text { USA: Alaska: Glacier } \\
\text { Bay National Park } \\
\text { (DO) }\end{array}$ & $\begin{array}{l}\text { Video of bears foraging in intertidal } \\
\text { zone by flipping rocks, searching } \\
\text { for and consuming crabs }\end{array}$ & N. Folsom (pers. comm.) \\
\hline \multirow[t]{12}{*}{$\begin{array}{l}\text { Raccoon } \\
\text { (Procyon lotor) A:35 }\end{array}$} & $\mathrm{N}$ & $\begin{array}{l}\text { USA: Connecticut: New } \\
\text { London: Bluff Point (DO) }\end{array}$ & $\begin{array}{l}\text { Bivalvia (Geukensia } \\
\text { demissa, Mya arenaria) }\end{array}$ & $\begin{array}{l}\text { Daiber (1982) } \\
\text { J. T. Carlton (pers. obs.) }\end{array}$ \\
\hline & $\mathrm{N}$ & USA: Gulf of Mexico & Brachyura (Uca pugilator) & Rathbun (1918) \\
\hline & $\mathrm{N}$ & $\begin{array}{l}\text { Pacific coast of America: } \\
\text { Puget Sound to Baja } \\
\text { California }\end{array}$ & $\begin{array}{l}\text { 'Foraging in the mussel beds'; 'visits } \\
\text { the shore at low tide feeding principally } \\
\text { on crabs' [Brachyura] }\end{array}$ & Ricketts et al. (1986) \\
\hline & I & $\begin{array}{l}\text { Canada: Queen } \\
\text { Charlotte Islands (S) }\end{array}$ & $\begin{array}{l}\text { Bivalvia (mussels); Gastropoda ('snail' and } \\
\text { 'limpet'); Cirripedia (Pollicipes polymerus); } \\
\text { Amphipoda; Isopoda; Brachyura (Hemi- } \\
\text { grapsus nudus); Echinoidea ('sea urchin') }\end{array}$ & Hartman $(1990,1993)$ \\
\hline & I & $\begin{array}{l}\text { Canada: Queen } \\
\text { Charlotte Islands }\end{array}$ & Foraging for small fish in intertidal zone & E. A. Mills (pers. comm.) \\
\hline & I & $\begin{array}{l}\text { Canada: Vancouver } \\
\text { Isl.: Bamfield (S) }\end{array}$ & Brachyura (Hemigrapsus) & D. Padilla (pers. comm.) \\
\hline & $\mathrm{N}$ & Washington & $\begin{array}{l}\text { Bivalvia (Mytilus trossulus); Brachyura (Hemi- } \\
\text { grapsus, nudus, H. oregonensis Cancer pro- } \\
\text { ductus); Anomura (Petrolisthes eriomerus); }\end{array}$ & - Dalquest (1948) \\
\hline & $\mathrm{N}$ & USA: Pacific coast & $\begin{array}{l}\text { Amphipoda (Talitridae, Megalorchestia } \\
\text { californiana) }\end{array}$ & Barnard et al.(1980) \\
\hline & $\mathrm{N}$ & $\begin{array}{l}\text { USA: California: Mendocino } \\
\text { Co.: Big River }\end{array}$ & $\begin{array}{l}\text { Bivalvia ('clams'); Brachyura ('crabs'); } \\
\text { Fish }\end{array}$ & Warrick \& Wilcox (1981) \\
\hline & $\mathrm{N}$ & $\begin{array}{l}\text { USA: California: Bodega } \\
\text { Bay: Bodega Head (S) }\end{array}$ & $\begin{array}{l}\text { Cirripedia (Pollicipes polymerus); Amphipoda } \\
\text { (Talitridae, Megalorchestia); Brachyura } \\
\text { (Pachygrapsus crassipes) }\end{array}$ & J. T. Carlton (pers. obs.) \\
\hline & $\mathrm{N}$ & $\begin{array}{l}\text { USA: California: Bodega } \\
\text { Bay: Bodega Harbor (S) }\end{array}$ & Brachyura (Carcinus maenas) & J. T. Carlton (pers. obs.) \\
\hline & $\mathrm{N}$ & USA: California: Pt. Reyes (S) & Anomura (Emerita analoga) & A. Cohen (pers. comm.) \\
\hline
\end{tabular}

(Table continued on next page) 
Table 1 (continued)

\begin{tabular}{|c|c|c|c|c|}
\hline Order/Species/ $/ \sum \mathrm{P}$ & N/I & Location/(observations) & Prey & Source \\
\hline & $\mathrm{N}$ & $\begin{array}{l}\text { USA: California: } \\
\text { Bolinas Lagoon (S) }\end{array}$ & Brachyura (Pachygrapsus crassipes) & T. Grosholz (pers. comm.) \\
\hline & $\mathrm{N}$ & USA: California: Santa Cruz (S) & Brachyura (Pachygrapsus crassipes) & R. Everett (pers. comm.) \\
\hline & $\mathrm{N}$ & $\begin{array}{l}\text { USA: Oregon: } \\
\text { Coos Bay (S, DO) }\end{array}$ & $\begin{array}{l}\text { Bivalvia (Mya arenaria Macoma nasuta, } \\
\text { Cryptomya californica); Brachyura (Cancer } \\
\text { magister); Anomura (Neotrypaea californiensis, } \\
\text { Upogebia pugettensis) }\end{array}$ & Davidson (1990) \\
\hline & $\mathrm{N}$ & $\begin{array}{l}\text { USA: Oregon: } \\
\text { Cape Blanco (DO) }\end{array}$ & Echinoidea (Strongylocentrotus purpuratus) & $\begin{array}{l}\text { J. Hodder \& J. T. Carlton } \\
\text { (pers. obs.) }\end{array}$ \\
\hline & $\mathrm{N}$ & $\begin{array}{l}\text { USA: Washington: } \\
\text { Willapa Bay (ST) }\end{array}$ & $\begin{array}{l}\text { Polychaeta (Nereis); Bivalvia, Mytilidae } \\
\text { (presumably Mytilus), Ostreidae (Ostrea); } \\
\text { Anomura (Emerita analoga), additional crabs } \\
\text { reported as Ocypodidae, but this family does } \\
\text { not occur in Washington; Caridea (Crangon } \\
\text { nigricauda); Echiura (Urechis caupo); Fish } \\
\text { (Clevelandia ios, Leptocottus armatus) }\end{array}$ & Tyson (1950) \\
\hline & $\mathrm{N}$ & $\begin{array}{l}\text { USA: Washington: } \\
\text { San Juan Isl. (DO) }\end{array}$ & Bivalvia (Venerupis philippinarum) & V. Galluci (pers. comm.) \\
\hline \multirow[t]{11}{*}{$\begin{array}{l}\text { American mink } \\
\text { (Mustela vison) } \\
\text { A:47 }\end{array}$} & I & Iceland: Grindavik (S) & $\begin{array}{l}\text { Amphipoda; Isopoda (Ligia oceanica, Idotea } \\
\text { spp.), Brachyura (Carcinus maenas), Fish (Myxo- } \\
\text { cephalus scorpius, Pholis gunnelus, Ciliata } \\
\text { mustela, Cyclopterus humpus, Gasterosteus } \\
\text { aculeatus, Pollachius virens, Sebastes sp.) }\end{array}$ & Skirmisson (1979) \\
\hline & I & UK: Scotland: Ross Peninsula & $\begin{array}{l}\text { Isopoda (Ligia oceanica?); Brachyura (Carcinus } \\
\text { maenas, other crabs); Fish (Anguilla anguilla, } \\
\text { Salmo sp., Gasterosteus aculeatus, Lipophyrs } \\
\text { pholis, Pholis gunnelus, Ciliata mustela, Taurulus } \\
\text { bubalis, Gobius sp., Spinachia vulgaris, flatfish) }\end{array}$ & 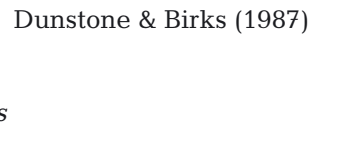 \\
\hline & I & Spain: Baiona & $\begin{array}{l}\text { Brachyura (Pachygrapsus marmoratus, Necora } \\
\text { puber, Carcinus maenas, Eriphia verrucosa); } \\
\text { Fish (Coryphoblennius galerita, Lipophrys } \\
\text { pholis, Gaidropsarus sp., Gobius cobitis) }\end{array}$ & Delibes et al. (2003) \\
\hline & $\mathrm{N}$ & USA: Atlantic coast & Forage at low tide on invertebrates & Amos (1966) \\
\hline & $\mathrm{N}$ & USA: Maine (DO) & Brachyura (Carcinus maenas) & D. Jacobs (pers. comm.) \\
\hline & $\mathrm{N}$ & USA: Connecticut: Noank (DO) & Foraging in intertidal zone: prey not known & D. A. Carlton (pers. comm.) \\
\hline & $\mathrm{N}$ & Canada: British Columbia (DO) & Brachyura ('crab') Echinoidea; 'shellfish' & Carefoot (1977) \\
\hline & $\mathrm{N}$ & Canada: British Columbia & Brachyura & Garth \& Abbot (1980) \\
\hline & $\mathrm{N}$ & USA: Alaska (southern) & $\begin{array}{l}\text { Bivalvia ('clams'), Brachyura ('crabs'), } \\
\text { 'other crustaceans'; fish ('tidepool') }\end{array}$ & O'Clair \& O'Clair (1998) \\
\hline & $\mathrm{N}$ & $\begin{array}{l}\text { USA: Washington: } \\
\text { San Juan Isl. (DO, S) }\end{array}$ & $\begin{array}{l}\text { Bivalvia (Veneroida, } 2 \text { spp.); Gastropoda (Lottidae, } \\
\text { Littorinidae, Trochidae); Mysidacea; Amphipoda; } \\
\text { Isopoda; Caridea (Hippolytidae); Brachyura } \\
\text { (Cancer; Majidae); Fish (Agonidae, Clupeidae, } \\
\text { Cottidae, Cyclopteridae, Gadidae, Pholididae, } \\
\text { Pleuronectiformes, Zoarcidae, Scorpaenidae, } \\
\text { Stichaeidae) }\end{array}$ & Perry (1998) \\
\hline & $\mathrm{N}$ & $\begin{array}{l}\text { USA: Oregon: } \\
\text { Cape Arago (DO) }\end{array}$ & Fish: sculpin in intertidal mussel beds & J. Jansen (pers. comm.) \\
\hline $\begin{array}{l}\text { American pine } \\
\text { marten (Martes } \\
\text { americana) A:1 }\end{array}$ & $\mathrm{N}$ & USA: Alaska (southern) & Marine crustaceans & O'Clair \& O'Clair (1998) \\
\hline $\begin{array}{l}\text { Old world badger } \\
\text { (Meles meles) A:2 }\end{array}$ & $\mathrm{N}$ & $\begin{array}{l}\text { Ireland: Fenit Is County } \\
\text { Kerry (DO \& S) }\end{array}$ & Amphipoda (Talitridae) Isopoda & $\begin{array}{l}\text { J. Davenport (pers. comm.) } \\
\text { Sleeman et al. (2001) }\end{array}$ \\
\hline $\begin{array}{l}\text { Striped skunk } \\
\text { (Mephitis mephitis) }\end{array}$ & N & USA: Atlantic coast & $\begin{array}{l}\text { Forage at low tide on invertebrates; 'ghost } \\
\text { crabs' (Brachyura: Ocypodidae) }\end{array}$ & Amos (1966) \\
\hline A: 2 & $\mathrm{~N}$ & USA: Washington: Puget Sound & Brachyura (Hemigrapsus nudus) & Dalquest (1948) \\
\hline Skunk (unidentified) & ) $\mathrm{N}$ & USA: Gulf of Mexico & Brachyura (Uca pugilator) & Rathbun (1918) \\
\hline $\begin{array}{l}\text { Domestic cat } \\
\text { (Felis catus) A:5 }\end{array}$ & I & USA: Gulf of Mexico & Brachyura (Uca pugilator) & Rathbun (1918) \\
\hline
\end{tabular}


Table 1 (continued)

\begin{tabular}{|c|c|c|c|c|}
\hline Order/Species/¿P & N/I & Location/(observations) & Prey & Source \\
\hline & I & USA: Gulf of Mexico & $\begin{array}{l}\text { 'Living among jetty rocks and feeding upon the } \\
\text { sessile mid-littoral jetty fauna' }\end{array}$ & Britton \& Morton (1989) \\
\hline & I & $\begin{array}{l}\text { Seychelles: } \\
\text { Aldabra Atoll (S, ST) }\end{array}$ & Brachyura (Grapsus tenicrustatus); Fish & Seabrook (1990) \\
\hline & I & Galapagos Islands & $\begin{array}{l}\text { Brachyura (Sally Lightfoot Crabs, } \\
\text { Grapsus grapsus); Isopoda }\end{array}$ & Lever (1994) \\
\hline \multicolumn{5}{|l|}{ Artiodactyla } \\
\hline \multirow[t]{5}{*}{$\begin{array}{l}\text { Domestic pig (Sus } \\
\text { scrofa) A:6; P:1 }\end{array}$} & I & $\begin{array}{l}\text { USA: Rhode Isl.: } \\
\text { Narragansett Bay (DO) }\end{array}$ & $\begin{array}{l}\text { Bivalvia (Mya arenaria or Mercenaria mer- } \\
\text { cenaria; oysters, Crassostrea virginica) }\end{array}$ & $\begin{array}{l}\text { Miantonomo (1642) } \\
\text { (in Cronon 1983) }\end{array}$ \\
\hline & I & USA: Oregon: Coos Bay & Bivalvia (Mya arenaria) & Peterson \& Powers (1952) \\
\hline & I & $\begin{array}{l}\text { New Zealand: Auckland } \\
\text { Islands (S of feral pigs) }\end{array}$ & Phaeophyta (Durvillea antarctica) & Chimera et al. (1995) \\
\hline & I & Tokelau Islands: Fakaofo & Cephalopoda ('octopi'); Fish ('eels') & Hyman (1999) \\
\hline & I & Peru (DO) & Anomura (Emerita) & J. P. Myers (pers. comm.) \\
\hline $\begin{array}{l}\text { Red deer (Cervus } \\
\text { elaphus) P:6 }\end{array}$ & $\mathrm{N}$ & UK: Isle of Rum (DO) & $\begin{array}{l}\text { Phaeophyta (Pelvetia caniculata, Fucus serratus, } \\
\text { F. spiralis, F. vesiculosus; Laminaria spp.) } \\
\text { Rhodophyta (Porphyra sp.) }\end{array}$ & Conradt (2000) \\
\hline \multirow[t]{5}{*}{$\begin{array}{l}\text { Mule (black-tailed) } \\
\text { deer (Odocoileus } \\
\text { hemionus) P:4 }\end{array}$} & I & $\begin{array}{l}\text { Canada: Queen } \\
\text { Charlotte Islands (DO) }\end{array}$ & $\begin{array}{l}\text { Chlorophyta (Ulva), Phaeophyta } \\
\text { (Nereocystis), and other algae }\end{array}$ & $\begin{array}{l}\text { T. Gaston (pers. comm.), } \\
\text { J. P. Myers (pers. comm.), } \\
\text { E. A. Mills (pers. comm.) }\end{array}$ \\
\hline & $\mathrm{N}$ & $\begin{array}{l}\text { USA: Alaska: Tsongas } \\
\text { National Forest (DO) }\end{array}$ & Algae & D. Cline (pers. comm.) \\
\hline & $\mathrm{N}$ & USA: Alaska (southern) & Phaeophyta (Alaria marginata) & O'Clair \& O'Clair (1998) \\
\hline & $\mathrm{N}$ & $\begin{array}{l}\text { USA: Washington: } \\
\text { San Juan Isl. }\end{array}$ & $\begin{array}{l}\text { Rhodophyta (intertidal) } \\
\text { Chlorophyta (Ulva) }\end{array}$ & $\begin{array}{l}\text { M. Herko (pers. comm.) } \\
\text { D. Padilla (pers. comm.) }\end{array}$ \\
\hline & $\mathrm{N}$ & $\begin{array}{l}\text { USA: California: } \\
\text { Bodega Bay: Bodega } \\
\text { Harbor (DO) }\end{array}$ & Chlorophyta? (Ulva?) & J. T. Carlton (pers. obs.) \\
\hline $\begin{array}{l}\text { Reindeer (Rangifer } \\
\text { tarandus) P:1 }\end{array}$ & I & South Georgia (ST) & Seaweed & Leader-Williams (1988) \\
\hline \multirow[t]{2}{*}{$\begin{array}{l}\text { Domestic goat } \\
\text { (Capra hircus) } \\
\text { P:3 }\end{array}$} & I & $\begin{array}{l}\text { USA: Washington: } \\
\text { Wadda Isl. (near Neah } \\
\text { Bay) (DO of feral goats) }\end{array}$ & Chlorophyta (Ulva sp.) & D. Padilla (pers. comm.) \\
\hline & I & $\begin{array}{l}\text { New Zealand: Auckland } \\
\text { Isl. (ST of feral goats) }\end{array}$ & $\begin{array}{l}\text { Phaeophyta (Macrocystis sp., Durvillea } \\
\text { antarctica) }\end{array}$ & Chimera et al. (1995) \\
\hline Striped skunk & $\mathrm{N}$ & USA: Atlantic coast & Forage at low tide on algae & Amos (1966) \\
\hline $\begin{array}{l}\text { Mountain goat } \\
\text { (Oreamnos } \\
\text { americanus) P:1 }\end{array}$ & $\mathrm{N}$ & $\begin{array}{l}\text { USA: Alaska: Tracy Arm } \\
\text { (South of Juneau) and Gla- } \\
\text { cier Bay National Park (DO) }\end{array}$ & (Fucus) in winter months & N. Folsom (pers. comm.) \\
\hline \multirow[t]{4}{*}{$\begin{array}{l}\text { Domestic sheep } \\
\text { (Ovis aries) P:4 }\end{array}$} & I & Iceland (DO) & $\begin{array}{l}\text { Rhodophyta (Rhodymenia); } \\
\text { Phaeophyta (Alaria) }\end{array}$ & Levring(1977) \\
\hline & I & $\begin{array}{l}\text { UK: Channel Islands: } \\
\text { Lihou (DO) }\end{array}$ & Phaeophyta (kelp) & Jarvis (1998) \\
\hline & I & UK: Orkney Isl. & Phaeophyta: (Laminariales; Fucus) & Hall (1975) \\
\hline & I & USA: Maine islands (DO) & Rhodophyta & P. Conkling (pers. comm.) \\
\hline
\end{tabular}

America), of which 4 are unique (Andean rat, house mouse, Pygmy rice rat, Peruvian desert fox). Together these 3 regions account for $42 \%$ of the diversity of maritime mammals recorded here.

The Eastern North Atlantic Ocean (Europe and Iceland) has 7 records ( 3 unique: white shrew, badger, red deer). There are 5 records from New Zealand and Australia, of which 2 are unique (Tasmanian devil and false water rat). Four unique records come from South
Africa: chacma baboon, rabbit, blackbacked jackal, and brown hyena.

For many other areas of the world, there are relatively few records, which we suggest below is due to underreporting. No records are in hand from Southeast Asia, and there are few reports from the Western South Atlantic Ocean (the east coast of South America) and the Mediterranean Sea (where, however, the intertidal zone is minimal to non-existent). 
Table 2. Terrestrial mammals feeding in the intertidal zone: summary of ordinal and species diversity

\begin{tabular}{|lc|}
\hline Order & No. of mammal species \\
\hline Didelphimorphia & 1 \\
Dasyuromorphia & 1 \\
Insectivora & 2 \\
Primates & 1 \\
Lagomorpha & 1 \\
Rodentia & 12 \\
Carnivora & 20 \\
Artiodactyla & 7 \\
Total orders: 8 & Total species: 45 \\
\hline
\end{tabular}

\section{Predator-prey diversity}

Of those prey identifiable to the level of phylum or lower (as opposed to prey only identified as 'intertidal organisms'), 228 different prey taxa are known to be consumed, representing 12 phyla of marine organisms (8 animal phyla, 3 protistan phyla [algae], and 1 plant phylum) (Table 3$)$. Two-thirds of these taxa $(67 \%$, or 153 taxa) are bivalve and gastropod mollusks, crabs, and fish (we discuss below potential biases that have entered into these taxa being recorded as dominant prey items).

Globally, no fewer than 26 maritime mammal species prey on intertidal crabs, 15 mammals prey on algae, 13 on bivalves, 12 on gastropods, and 11 on fish (Table 3). Eight maritime mammals consume only plant or algal prey (mule deer, red deer, reindeer, goat, mountain goat, sheep, rabbit, and the Beechey ground squirrel); 28 maritime mammal species consume only animal prey, and only 7 mammals are known to consume both animal and plant prey (grass mouse, house mouse, Norway rat, rice rat, coyote, Arctic fox, and black bear) (Table 1). The prey of 2 species (Keen's mouse and Townsend's vole) is as yet unknown.

\section{Non-indigenous predators and non-indigenous prey}

Introduced populations of 17 species of mammals have been recorded as maritime predators (Table 1). Fifteen of these species are recorded on islands where endemic mammals were absent or rare, making predation by introduced mammals perhaps more obvious.

In turn, 4 maritime mammals utilize, in part, non-native prey. The false water rat in Australia, the raccoon in California, and the mink in Maine all prey on introduced populations of the European shore crab Carcinus maenas. On the Pacific coast, raccoons prey upon the introduced Atlantic clam Mya arenaria and the introduced Japanese clam Venerupis philippinarum.
In only one instance is there a record (from Oregon) of an introduced mammal (pigs) preying upon introduced prey (the Atlantic clam Mya arenaria).

\section{DISCUSSION}

\section{Biogeography and diversity of maritime mammals: biases}

Data biases and limitations obviously attend this global review of maritime mammals. The most overwhelming bias is a geographic one. Striking examples are now-cosmopolitan species such as the house mouse Mus musculus (which is likely a predator in many regions of the world, but for which there is but one report in the Eastern South Pacific Ocean, Chile), and the domestic rabbit Oryctolagus cuniculus (for which only South African intertidal records are available, but which likely invades the intertidal zone in many areas of the world). We suggest that the lack of reports of terrestrial mammal predation in intertidal

Table 3. Number of prey species consumed by maritime mammals

\begin{tabular}{|c|c|c|}
\hline & $\begin{array}{l}\text { No. of } \\
\text { prey } \\
\text { species }\end{array}$ & $\begin{array}{c}\text { No. of maritime } \\
\text { mammal predators } \\
\text { consuming prey type }\end{array}$ \\
\hline Porifera & 2 & 1 \\
\hline \multicolumn{3}{|l|}{ Annelida } \\
\hline Polychaeta & 5 & 4 \\
\hline Echiura & 1 & 1 \\
\hline \multicolumn{3}{|l|}{ Mollusca } \\
\hline Bivalvia & 30 & 13 \\
\hline Gastropoda & 40 & 12 \\
\hline Polyplacophora & 4 & 2 \\
\hline Cephalopoda & 1 & 1 \\
\hline \multicolumn{3}{|l|}{ Crustacea } \\
\hline Cirripedia & 5 & 5 \\
\hline Mysidacea & 1 & 2 \\
\hline Amphipoda & 7 & 12 \\
\hline Isopoda & 8 & 6 \\
\hline Caridea & 2 & 2 \\
\hline Brachyura/Anomura & 42 & 26 \\
\hline Astacura & 1 & 1 \\
\hline Insecta & 3 & 3 \\
\hline \multicolumn{3}{|l|}{ Echinodermata } \\
\hline Asteroidea & 3 & 3 \\
\hline Echinoidea & 4 & 5 \\
\hline Holothuroidea & 3 & 2 \\
\hline \multicolumn{3}{|l|}{ Urochordata } \\
\hline Ascidiacea & 2 & 1 \\
\hline Fish & 41 & 11 \\
\hline Algae & 22 & 15 \\
\hline Seagrass & 1 & 1 \\
\hline Total: & 228 & \\
\hline
\end{tabular}


communities in many areas of the world - even those well studied by marine ecologists - is not due to the rarity of the phenomenon. Rather, we hypothesize that many observations around the world have simply not been reported, or remain recorded in obscure places.

When there are reports, it is also clear that there are biases in the nature and quality of the data. Research biases or limited geographic data contribute strongly to any sense of those mammals with the highest diversity of prey. Thus, while Norway rats appear to consume more prey (66 taxa) than any other mammal, these data are derived largely from only 3 locations (Chile, Italy, and New Zealand). Similarly, data indicating that house rats eat 16 different taxa are derived almost entirely from Chile. Perhaps more realistic is the prey diversity of raccoons (35 taxa), representing observations along much of the Pacific coast of North America, and the prey diversity of mink (47 taxa), representing data from both the Pacific and Atlantic Oceans.

Furthermore, observations have largely been in the daytime - when hunting activity is likely to be at its lowest for these crepuscular and nocturnal predators. Most observations are largely incidental to other studies, and these in turn are seasonally biased (much intertidal work and observations being in the summer).

There are 5 sources for prey data. These are: (1) direct observation of prey consumption where the identity of the prey is known; (2) direct observation of predator foraging and live prey handling, without actual observation of specific prey ingested; (3) study of stomach contents; (4) study of scat (fecal) material; and (5) analysis of the contents of middens, burrows, and dens.

Challenges and complexities are associated with each of these approaches, but particularly with the indirect observations in the last 3 data sources. In the absence of direct observation (Sources 1 and 2) it may not be possible to determine if prey were consumed alive or scavenged. For example, black-backed jackal middens in Namibia contain fish and crabs, but it is not possible to determine whether these organisms were taken alive or scavenged dead (Avery et al. 1987). Relative to scat contents, a comparison of scats and direct observations of predation by black-backed jackals revealed considerable differences: scats included invertebrate prey, whereas direct observations did not (Hiscocks \& Perrin 1987). Similarly, it is not possible to determine via stomach contents whether ruminants are feeding on live attached algae or on drift algae washed ashore.

The potential role of the consumption of secondarily derived food is a further challenge when using indirect observations. Secondarily derived food is defined as food not directly or intentionally obtained alive by the predator. Secondary prey may be derived from 4 sources: (1) Scavenging: consumers may eat regurgitated food or food dropped by other predators. Black-backed jackals in Namibia feed on regurgitated fish dropped by cormorants (Avery et al. 1987) and also appear to feed on mussels Perna perna dropped by gulls (Nel \& Loutit 1986). The presence of fish in the stomachs of Norway rats on the Queen Charlotte Islands may be the result of the rats eating prey brought inland by bald eagles and other scavengers (Drever \& Harestad 1998). (2) Kleptoparasitism: Avery et al. (1987) speculate that the blackbacked jackals noted by Nel \& Loutit (1986) may have actively stolen the clams from the gulls, rather than picking up remnants. Black-backed jackals are known to rob gulls of the clam Donax serrata, freshly dropped by gulls on hard surfaces such as rocks and roads (Hiscocks \& Perrin 1987). (3) Consumption of epibiota or endobiota (organisms living on or in the target prey organism): Norway rats eating the intertidal black mussel Xenostrobus also consume the mussel's endocommensal pea crab Pinnotheres, as well as organisms living on the mussel's shell, such as the barnacle Chamaesipho and the tiny gastropod Eatoniella (Moors 1985). (4) Consumption of the prey's stomach contents: mink in Scotland were found to have amphipods in their stomach, but these may have been derived, in turn, from the stomachs of ingested fish prey (Dunstone \& Birks 1987). Mink in Spain were similarly found to have small mollusks in their feces, but these appear to be the prey of the intertidal fish upon which the mink had fed (Delibes et al. 2003).

\section{Why would terrestrial mammals utilize the intertidal zone as an energy resource?}

There are at least 3 alternative scenarios - over a spectrum of spatial and temporal conditions - as to why terrestrial mammals would intentionally seek out and utilize resources in the intertidal zone.

(1) Facultative omnivory: some mammal species may simply be opportunistic omnivores. Indeed, many records of single observations of predator episodes for many of the mammals shown in Table 1 may simply be facultative omnivory. (2) Perennial trophic subsidies: mammals that live in perennially impoverished nearcoastal ecosystems may seek out the adjacent and potentially more productive marine ecosystem for food. In the arid deserts adjacent to the Gulf of California, coyotes Canis latrans feed on a variety of marine organisms, including sea turtles, marine mammals, fish, birds, invertebrates, and algae (Rose \& Polis 
1998). Dogs on impoverished Canton Atoll (Kiribati) learned to herd fish into shallow water (Degener \& Gillaspy 1955). (3) Seasonal trophic subsidies: mammals that live in seasonally or periodically impoverished near-coastal ecosystems may also seek out adjacent productive marine resources. Numerous examples are available. Deer in Alaska come down to the shore in winter to feed on intertidal algae (D. Cline pers. comm.; O'Clair \& O'Clair 1998). Marine invertebrate use increases in the winter in Arctic fox diets on St. Lawrence Island in the Bering Sea (Fay \& Stephenson 1989). On islands off Baja California, the deer mouse Peromyscus maniculatus is a granivore in wet years, but feeds extensively on intertidal organisms in dry years, as revealed by stable isotope analysis (Stapp et al. 1999, Stapp 2002). On a British Columbia island, Keen's mice Peromyscus keeni appear to expand their diet to marine invertebrates before seabird eggs become available, which is work also based on stable isotope analysis (Drever et al. 2000). Feral rabbits in South Africa are believed to feed on marine algae to survive dry summers (Moore 2002). The increase in fish predation in winter by mink in Scotland may be the result of decreased availability of their rabbit prey (Dunstone \& Birks 1987). Red fox, presumably in the state of Washington, foraged intertidally in the spring and summer (May to July), but then decreased intertidal predation in August when terrestrial food (blackberries) became available (Holmes 1992). Avery \& Siegfried (1980) implied that a seasonal effect may also regulate, in part, Chacma baboon intertidal predation in South Africa, noting that the baboons 'are known to capture and consume marine shellfish and crustaceans, especially when the protein content of their normal terrestrial plant food is low'. The numerous reports (Table 1) of bears feeding intertidally in Alaska and British Columbia may further reflect annual or periodic depression of their terrestrial food resources.

Alternatively, it may be noted that for some predators, food hoarding may alleviate the need to forage intertidally. Red and Arctic foxes thus store prey, particularly seabird carcasses, in the winter (Fay \& Stephenson 1989, Sklepkovych \& Montevecchi 1996), but storage of marine invertebrates as winter food resources has not yet been reported.

\section{What are the ecological and evolutionary consequences of maritime mammal predation?}

What evidence is there that terrestrial mammal predation (1) influences the diversity, abundance or distribution of prey species (and thus has the potential to impact community structure), and (2) is important in the landward flow of energy (and thus has the poten- tial to influence the abundance and distribution of predator species)?

The lack of experimental data (discussed below as a future key direction in maritime mammal research) inhibits any extensive conclusions on the role maritime mammals may play in regulating community structure. We note that predators need not necessarily be abundant to affect an impact on prey distribution and abundance, as Frank (1982) has demonstrated relative to oystercatcher predation on intertidal limpets, and as Estes et al. (1998) have shown relative to killer whale predation on sea otters. Wooton (1992) has suggested that raccoons preying on sea urchins (Strongylocentrotus spp.) on the mainland coast of Washington 'may have an impact upon sea urchins comparable to birds'. At these Washington sites gulls, black oystercatchers, and crows had important impacts on the abundance and distribution of sea urchins in low intertidal communities, effects which then cascaded down to indirectly affecting both the abundance and diversity of macroalgae (Wooton 1992).

The potentially most important impact of Norway rat predation in the Chilean intertidal is on the size and abundance of keyhole limpets (Navarrete \& Castilla 1993). Rats fed on the smaller and less abundant size classes of 2 species of these limpets - potentially removing nearly $20 \%$ of the small limpets annually in the region studied. Keyhole limpets (like sea urchins) may in turn control the abundance of algae (Navarrete \& Castilla 1993), and thus rat predation on limpets could have an important indirect impact on algal distribution and biomass. Mink in Spain (at a population density of $4 \mathrm{mink} \mathrm{km}^{-1}$ ) are estimated to consume 3700 blennies and 1850 crabs $\mathrm{km}^{-1}$ of shoreline in August; a single mink may consume 110 to $147 \mathrm{~g}$ of fish (26 to 35 ind.) and 65 to $139 \mathrm{~g}$ of crabs (10 to 22 ind.) (Delibes et al. 2003). In turn, there may be critical cascading impacts of the predator-mediated removal of this number of fish and decapods on the blennies' and crabs' prey.

Where predation intensity by terrestrial mammals on the shore is consistent and high, maritime mammals may further be able to set the upper intertidal limit of certain prey. Sheep, deer, and goats feeding on upper shore algae may be important in this regard, and exclusion experiments are called for. Island algal populations that have sustained decades or centuries of feeding pressure by sheep (Moore 2002) would be of particular interest in this regard.

Data are in hand, however, on the importance of landward flow of energy from the sea to the land (Polis \& Hurd 1996a,b, Polis et al. 1997, Anderson \& Polis 1998, Huxel \& McCann 1998, Rose \& Polis 1998). Rose \& Polis (1998) demonstrated that in the arid deserts of Baja California, coyote Canis latrans abundance along 
the shore is 2.4 to 13.7 times higher than adjacent inland areas, densities achieved (as noted above) by consumption of marine organisms. Scat mass was more than double at coastal locations compared to inland study sites, and nearly half of all items in coastal scats were from the ocean. West (1987) found that Arctic fox populations on the Aleutian Islands 'appear to be buffered from starvation by the abundance and renewable nature of coastal marine resources'. Stewart et al. (1989) suggested that the high densities and extended breeding season of masked shrews Sorex cinereus on a Nova Scotia island were sustained by abundant littoral prey, particularly amphipods and kelp flies. Thus in certain biomes - impoverished coastal land systems and islands, for example - the utilization of shore energy by predators may be critical in permitting the success of species in regions that would not otherwise support large or viable populations of these mammals.

The significance of introduced predators, or of introduced prey, in maritime mammal ecology largely remains to be explored. Introduced mammals on islands which previously had no mammals have added a new guild of intertidal predators to such regions. In certain regions introduced mammal populations that prey on the shore may have been enhanced by their general proximity to urban areas. Agricultural practices may sustain relatively large populations of introduced domestic grazing animals (such as pigs, sheep, and goats) in rural areas adjacent to intertidal shores. In general, one largely unrecognized aspect of mammalian invasions may be their impact on marine intertidal communities. Such impact may have long preceded the arrival of the first ecologists. For example Rattus exulans was brought to New Zealand ca. 1000 yr ago by Maoris and $R$. norvegicus ca. $400 \mathrm{yr}$ ago by Europeans (Moors 1985), and thus may have been intertidal predators in these regions for centuries. In many areas of the world, populations of marine animals preyed on by introduced mammals may have undergone modifications well before any scientific studies were launched.

Ecological and evolutionary consequences of maritime mammal predation may include the potential for intraspecific specialization. Individual Norway rats preying in the Chilean intertidal zone show some degree of specialization (Navarrete \& Castilla 1993). Rats in Italy dig and dive for mollusks at low tide, suggesting individual specialization has occurred here as well (Parisi \& Gandolfi 1974). Coastal mink diets in Scotland may vary individually (perhaps partly as mediated by individual home range), as well as by gender (larger male mink prey more heavily on rabbits, whereas smaller females prey more heavily on fish and crustaceans) (Dunstone \& Birks 1987). Mink in Spain use core (critical) areas within a home range, and in coastal regions such areas include the intertidal zone (Bonesi et al. 2000). Conradt (2000) found that adult sons and daughters of red deer on the Isle of Rum, Scotland, adopted their mother's extent of feeding on seaweed and thus learned to use the seaweed habitat early in life, suggesting the potential for specialization within the species.

\section{Conclusion: Is terrestrial mammal predation in the intertidal a rare phenomenon, a highly localized phenomenon, or common but overlooked (and thus rarely studied)?}

We posed at the beginning of this review 3 scenarios that could account for the lack of recognition and attention paid to the phenomenon of terrestrial mammals visiting the shore: (1) Predation on marine organisms by terrestrial mammals may be rare everywhere. Our data suggest that this is not the case: maritime mammals appear to be widespread globally, with often repeated cases of predation being observed by the same mammal species; (2) Predation on marine organisms by terrestrial mammals may occur only in certain localized regions, albeit perhaps common at such sites. Our data suggest that this is also not the case, although as we have discussed earlier, because of those processes that could draw mammals to the shore, there may be areas where this phenomenon is relatively more important, even if globally ubiquitous; (3) Predation on marine organisms by terrestrial mammals is relatively common but largely underreported and overlooked. We suggest that this conclusion-that predation by maritime mammals is a rarely studied phenomenon rather than a rare phenomenon - bears careful consideration, based on the abundance of observations across a wide variety of taxa (Table 1).

What is now required, however, to determine the importance of maritime mammal predation are 2 categories of data: (1) quantitative observations on predation, and (2) experimental studies. Field observations and data collection could and should be facilitated by the use of modern technologies, including advanced infrared night vision technology, radiotelemetry of individuals (Birks \& Dunstone 1985, Millspaugh \& Marzluff 2001, Moore 2002), and the use of stable isotopes for trophic web analysis (Angerbjorn et al. 1994, Stapp et al. 1999, Drever et al. 2000, Stapp 2002). Experimental studies on maritime mammals (noted by Navarrete \& Castilla 1993 as an important challenge) could be conducted by the use of large exclosures, or in areas where the removal of introduced mammals is proposed, such as on islands (Wood et al. 2002). Particularly worthy of experimental work would be maritime mammal populations for which some quantitative prey data already exist, such as the Norway rat populations 
in Chile and Arctic fox populations on certain Aleutian Islands. Raccoon populations would also appear to be particularly amenable for experimental manipulative studies. Locating comparable communities with and without maritime mammal predators may provide further insight, such as nearshore islands without raccoons facing a mainland with raccoons acting as known maritime mammals. Lacking experimental evidence, the scale of interactions - whether strong, weak, or insignificant - cannot be adequately assessed (Menge \& Sutherland 1987).

We conclude by expanding upon the conclusions of Stapp et al. (1999): the importance of intertidal resources in supplying energy to terrestrial populations of many species of mammals world wide may have been underappreciated. Maritime mammals have been a largely overlooked guild of intertidal predators. Where populations and individuals of certain species are marine dependent, the impact on the abundance, diversity, and distribution of intertidal prey — and thus the implications for the diversity of trophic networks and cascades in marine coastal ecosystems-may have been similarly underappreciated.

Acknowledgements. We thank G. Branch, D. Carlton, D. Cline, P. Conkling, A. Cohen, J. Davenport, L. Eldredge, R. Everett, N. Folsom, V. Galluci, T. Gaston, M. Graybill, C. Griffiths, T. Grosholz, M. Herko, C. Hewitt, D. Jacobs, J. Jansen, P. Jokiel, T. Klinger, M. Matthews, E. A. Mills, P. G. Moore, P. Myers, D. Padilla, G. Parry, and N. Seto for providing original observations and suggestions. We thank B. Butler (OIMB Librarian) for aiding in obtaining literature, and C. Mills for interviewing D. Padilla, E. A. Mills, T. Klinger and M. Herko.

\section{LITERATURE CITED}

Amos WH (1966) The life of the seashore. McGraw-Hill Book Company, Toronto

Anderson WB, Polis GA (1998) Marine subsidies of island communities in the Gulf of California: evidence from stable carbon and nitrogen isotopes. Oikos 81:75-80

Angerbjorn A, Hersteinsson P, Liden P, Nelson E (1994) Dietary variation in arctic foxes (Alopex lagopus) - an analysis of stable carbon isotopes. Oecologica 99:226-232

Avery G, Siegfried WR (1980) Food gatherers along South Africa's seashore. Oceans 13:37

Avery G, Avery DM, Braine S, Loutit R (1987) Prey of coastal black-backed jackal Canis mesomelas (Mammalia: Canidae) in the Skeleton Coast Park, Namibia. J Zool Lond 213:81-94

Barnard JL, Bowers D, Bousfield EL (1980) Amphipoda: The amphipods and allies. In: Morris RH, Abbott DP, Haderlie EC (eds) Intertidal invertebrates of California. Stanford University Press, Stanford, p 559-566

Birks JDS, Dunstone N (1985) Sex related differences in the diet of the mink, Mustela vison. Holarc Ecol 8:245-252

Bonesi L, Dunstone N, O'Connell M (2000) Winter selection of habitats within intertidal foraging areas of mink (Mustela vison). J Zool Lond 250:419-424

Braestrup FW (1941) A study on the arctic fox in Greenland: immigrations, fluctuations in numbers based mainly on trading statistics. Medd Gronl 131:1-101

Britton JC, Morton B (1989) Shore ecology of the Gulf of Mexico. University of Texas Press, Austin

Brown AC, McLachlan A (1990) Ecology of sandy shores. Elsevier, Amsterdam

Campbell RR (1988) Status of the sea mink, Mustela macrodon, in Canada. Can Field Nat 102:304-306

Carefoot T (1977) Pacific seashores, a guide to intertidal ecology. University of Washington Press, Seattle

Carlton JT, Geller JB, Reaka-Kudla ML, Norse EA (1999) Historical extinctions in the sea. Annu Rev Ecol Syst 30: 515-538

Chimera C, Coleman MC, Parkes JP (1995) Diet of feral goats and feral pigs on Auckland Island, New Zealand. NZ J Ecol 19:203-207

Churchfield S (1990) The natural history of shrews. Christopher Helm, London

Conkling PW (1999) Islands in time. A natural history and cultural history of the islands of the Gulf of Maine. Island Institute, Rockland Maine and Down East Books, Camden, $\mathrm{ME}$

Conradt L (2000) Use of a seaweed habitat by red deer (Cervus elaphus L.). J Zool 250:541-549

Crawford C (1953) Coyotes dig clams and fish squeal in Santa Maria Bay. Los Angeles Times, Los Angeles. Reprinted (September 1953) in the Minutes of the Conchological Club of Southern California

Crawford RJM, Shelton PA (1978) Pelagic fish and seabird interrelationships off the coasts of south west and South Africa. Biol Conserv 14:85-109

Cronon W (1983) Changes in the land. Indians, colonist, and the ecology of New England. Hill and Wang, New York

Crosby AWJ (1972) The Columbian exchange: biological and cultural consequences of 1492. Greenwood Press, Westport, CT

Daiber FC (1982) Animals of the tidal marsh. Van Nostrand Reinhold, New York

Dalquest WW (1948) Mammals of Washington. Univ Kans Mus Nat Hist Publ 2:1-444

Davidson SK (1990) Raccoon foraging in the tidal flats of the Coos Estuary. Honors College BA thesis, Department of Biology, University of Oregon, Eugene

Degener O, Gillaspy E (1955) Canton Island. Atoll Res Bull 41: 51

Delibes M, Clavero M, Prenda J, Blazquez C, Ferreras P (2003) A terrestrial mammal invades intertidal communities: the American mink (Mustela vison) in southern Europe. Biol Invas 5 (in press)

Drever MC, Harestad AS (1998) Diets of Norway rats, Rattus norvegicus, on Langara Island, Queen Charlotte Islands, British Columbia: implications for conservation of breeding seabirds. Can Field-Nat 112:676-683

Drever MC, Blight LK, Hobson KA, Bertram DF (2000) Predation on seabird eggs by Keen's mice (Peromyscus keeni): using stable isotopes to decipher the diet of a terrestrial omnivore on a remote offshore island. Can J Zool 78: 2010-2018

Dunstone N, Birks JDS (1987) The feeding ecology of mink (Mustela vison) in coastal habitat. J Zool 212:69-83

Ellis DW, Wilson S (1981) The knowledge and usage of marine invertebrates by the Skidegate Haida people of the Queen Charlotte Islands. Queen Charlotte Islands Museum Society. Monograph Series 1, Skidegate, BC

Estes JA, Tinker T, Williams TM, Doak D (1998) Killer whale predation on sea otters linking oceanic and nearshore ecosystems. Science 282:472-475 
Fay FH, Stephenson RO (1989) Annual, seasonal, and habitatrelated variation in feeding habits of the arctic fox (Alopex lagopus) on St. Lawrence Island, Bering Sea. Can J Zool 67:1986-1994

Fox MW (1975) The wild canids: their systematics, behavioral ecology and evolution. Robert E. Krieger Publishing Company, Malabar

Frank PW (1965) The biodemography of an intertidal snail population. Ecology 46:831-844

Frank PW (1982) Effects of winter feeding on limpets by Black Oystercatchers, Haematopus bachmani. Ecology 63: 1352-1362

Garth JL, Abbott DP (1980) Brachyura: the true crabs. In: Morris RH, Abbott DP, Haderlie EC (eds) Intertidal invertebrates of California. Stanford University Press, Stanford, p 594-630

Graham MH, Dayton PK, Erlandson JM (2003) Ice ages and ecological transitions on temperate coasts. Trends Ecol Evol 18:33-40

Hall KRL (1962) Numerical data, maintenance activities and locomotion of the wild chacma baboon, Papio ursinus. Proc Zool Soc Lond 139:181-220

Hall SJG (1975) Some recent observations on Orkney sheep. Mamm Rev 5:59-64

Hartman LH (1990) Raccoon-seabird interactions on the Queen Charlotte Islands: assessing the risk. Pac Seabird Group Bull 17:21

Hartman LH (1993) Ecology of coastal raccoons (Procyon iotor) on the Queen Charlotte Islands, British Columbia, and evaluation of their potential impact on native burrownesting seabirds. MSc thesis, University of Victoria, Canada

Heatwole H (1971) Marine-dependent terrestrial biotic communities on some cays in the Coral Sea. Ecology 52: 363-366.

Hersteinsson P, MacDonald DW (1996) Diet of arctic foxes (Alopex lagopus) in Iceland. J Zool Lond 240:457-474

Hirth HF (1987) Synopsis of biological data on the green turtle Chelonia midas (Linnaeus) (1758). US Fish Wildl Serv Biol Rep 97(1)

Hiscocks K, Perrin MR (1987) Feeding observations and diet of black-backed jackals in an arid coastal environment. S Afr J Wildl Res 17:53-58

Holmes RE (1992) Red fox use of the intertidal zone. Northwest Sci 66:115

Huey RB (1969) Winter diet of the Peruvian desert fox. Ecology 50:1089-1090

Huxel GR, McCann K (1998) Food web stability: the influence of trophic flows across habitats. Am Nat 152:460-469

Hyman B (1999) A rare trip to the Tokelaus. Pacific Magazine $24: 44-45$

Jarvis P (1998) Seagoing sheep. New Sci 51

Josselyn J (1674) An account of two voyages to New-England. Printed for Giles Widdows, London

Kapel CMO (1999) Diet of arctic foxes (Alopex lagopus) in Greenland. Arctic 52:289-293

King WB (1985) Island birds: Will the future repeat the past? ICBP Tech Pub 3:3-15

Krebs CJ (1993) Ecology: the experimental analysis of distribution and abundance. Harper Collins College Publishers, New York

Lavrov NP (1932) The arctic fox. In: Bureau of animal populations translation 18F, 1079A:1-92. Oxford University Press, England (translation by JD Jackson, 1940)

Leader-Williams N (1988) Reindeer on South Georgia. Cambridge University Press, Cambridge

Lever C (1994) Naturalized animals: the ecology of success- fully introduced species. T \& AD Poyser, London

Levinton JS (2001) Marine biology: function, biodiversity, ecology. Oxford University Press, Oxford

Levring T (1977) Potential yields of marine algae-with emphasis on European species. In: Krauss RW (ed) The marine plant biomass of the Pacific Northwest coast. Oregon State University Press, Corvallis, p 251-270

Manville RH (1966) The extinct sea mink, with taxonomic notes. Proc US Natl Mus 122 (3584):1-12

Martinez DR, Lopez DA, Del Villar ML, Figueroa FR, Gajardo HF (1986) Depredacion de organismos intermareales por roedores. Biota 2:87-90

Menge BA, Sutherland JP (1987) Community regulation: variation in disturbance, competition, and predation in relation to environmental stress and recruitment. Am Nat 130:730-737

Millspaugh JJ, Marzluff JM (eds) (2001) Radio tracking and animal populations. Academic Press, New York

Moore PG (2002) Mammals in intertidal and maritime ecosystems: interactions, impacts and implications. Oceanogr Mar Biol Annu Rev 40:491-608

Moors PJ (1985) Norway rats Rattus norvegicus on the Noises and Motukawa Islands, Hauraki Gulf, New Zealand. NZ J Ecol 8:37-54

Murie OJ (1959) Fauna of the Aleutian Islands and the Alaska Peninsula. North Am Fauna 61:1-406

National Geographic Society (1998) Tale of the African tides. National Geographic Explorer Film Series: www.nationalgeographic.com/tv/explorer/exp052800.html. National Geographic Society, Washington, DC

National Research Council (1990) Decline of the sea turtles: causes and prevention. National Academy Press, Washington, DC

Navarrete SA, Castilla JC (1993) Predation by Norway rats in the intertidal zone of Central Chile. Mar Ecol Prog Ser 92: 187-199

Nel JA, Loutit R (1986) The diet of black-backed jackals Canis mesomelas, on the Namib desert coast. Cimbebasia 8: 91-96

Nielsen SM (1991) Fishing arctic foxes Alopex lagopus on a rocky island in West Greenland. Polar Res 9:211-213

Novikov GA (1956) Carnivorous mammals of the fauna of the USSR. S. Monson, Jerusalem

Nowak RM (1999) Walker's mammals of the world. The John Hopkins University Press, London

Nybakken JW (2000) Marine biology: an ecological approach. Benjamin Cummings, Menlo Park, CA

O'Clair RM, O'Clair CE (1998) Southeast Alaska's rocky shores: animals. Plant Press, Auke Bay, AK

Oldershaw K (1995) An observational study of black bears on Jenny's Beach in Shelter Inlet. Report of the Clayoquot Biosphere Project, Tofino, British Columbia

Osborne TO, Sheppe WA (1971) Food habits of Peromyscus maniculatus on a California beach. J Mamm 52:844-845

Parisi V, Gandolfi G (1974) Further aspects of the predation by rats on various mollusc species. Boll Zool 4:87-106

Perry SL (1998) Prey items of mink, Mustela vison, in coastal areas along eastern San Juan Island: utilization of marine organisms by a terrestrial carnivore. Class report for Zoology 533a-Marine Conservation Biology, University of Washington, Friday Harbor Laboratories Library, Friday Harbor, WA

Peterson ER, Powers A (1952) A century of coos and curry: history of Southwestern Oregon. Binfords and Mort, Portland, OR

Polis GA, Hurd SD (1996a) Allochthonous input across habitats, subsidized consumers, and apparent trophic cas- 
cades: examples from the ocean-land interface. In: Polis GA, Winemiller KO (eds) Food webs: integration of patterns and dynamics. Chapman \& Hall, San Francisco

Polis GA, Hurd SD (1996b) Linking marine and terrestrial food webs: allochthonous input from the ocean supports high secondary productivity on small islands and coastal land communities. Am Nat 147:396-423

Polis GA, Anderson WB, Holt RD (1997) Toward an integration of landscape and food web ecology: the dynamics of spatially subsidized food webs. Annu Rev Ecol Syst 28: 289-316

Raffaelli D, Hawkins S (1996) Intertidal ecology. Chapman \& Hall, London

Ramsay CR (1985) The Northwest. In: Crump DJ (ed) America's seashore wonderlands. National Geographic Society, Washington, DC, p 10-43

Rathbun MJ (1918) The grapsoid crabs of America. US Nat Mus Bull 97:461

Ricketts EF, Calvin J, Hedgpeth JW (1952) Between Pacific tides. Stanford University Press, Stanford, CA

Ricketts EF, Calvin J, Hedgpeth JW, Phillips D (1986) Between Pacific tides. Stanford University Press, Stanford, CA

Ricklefs RE, Miller G (1999) Ecology. WH Freeman, New York

Roest AI (1993) Ground squirrels feeding on eelgrass. Calif Fish Game 79:85-86

Rose MD, Polis GA (1998) The distribution and abundance of coyotes: the effects of allochthonous food subsidies from the sea. Ecology 79:998-1007

Seabrook W (1990) The impact of the feral cat (Felis catus) on the native fauna of Aldabra Atoll, Seychelles. Rev Ecol (Terre Vie) 45:135-145

Shanholtzer GF (1974) Relationship of vertebrates to salt marsh plants. In: Reimold RJ, Queen WH (eds) Ecology of halophytes. Academic Press, New York, p 463-474

Skirmisson K (1979) Food habits of mink (Mustela vison Schreber) in Grindavik, south west Iceland. Natturufraedingurinn 39:194-203

Sklepkovych BO, Montevecchi WA (1996) Food availability and food hoarding behavior by red and arctic foxes. Arctic 49:228-234

Sleeman P, Cussen R, O'Donoughue T, Costello E (2001) Badgers (Meles meles) on Fenit Island, and their presence or absence on other islands in Co. Kerry, Iceland. Small Carniv Conserv 24:10-12

Stapp P (2002) Stable isotopes reveal evidence of predation by ship rats on seabirds on the Shiant Islands, Scotland. J Appl Ecol 39:831-840

Editorial responsibility: Otto Kinne (Editor),

Oldendorf/Luhe, Germany
Stapp P, Polis GA, Sanchez Pinero F (1999) Stable isotopes reveal strong marine and El Nino effects on island food webs. Nature 401:467-469

Stewart DT, Herman TB, Teferi T (1989) Littoral feeding in a high-density insular population of Sorex cinereus. Can J Zool 67:2074-2077

Strange IJ (1983) The Falkland Islands. David \& Charles, North Pomfret

Stuart CT, Shaughnessy PD (1984) Content of Hyaena brunnea and Canis mesomelas scats from southern coastal Namibia. Mammalia 48:611

Thompson RC, Jenkins SR, Bussell JA (2000) A method for recording predator-prey encounters between crabs and limpets using wax replicas. J Mar Biol Assoc UK 80: 633-638

Thorson G (1971) Life in the sea. McGraw-Hill Book Company, New York

Tyson EL (1950) Summer food habits of the raccoon in southwest Washington. J Mamm 31:448-449

Valiela I (1995) Marine ecological processes. Springer Verlag, Heidelberg

Vinogradov ME (1968) Vertical distribution of the oceanic zooplankton. Keter Press, Jerusalem

Warrick SF, Wilcox ED (1981) Big river. The natural history of an endangered northern California estuary. University of California, Santa Cruz

West EW (1987) Food habits of Aleutian Island arctic foxes. Murrelet 68:33-38

Willson MF, Gende SM, Marston BH (1998) Fishes and the forest. BioScience 48:455-462

Wolff T (1976) Utilization of sea grass in the deep sea. Aquat Bot 2:161-74

Wood B, Tershy BR, Hermosillo MA, Donlan CJ and 5 others (2002) Removing cats from islands in northwest Mexico. In: Veitch CR, Clout MN (eds) Turning the tide: the eradication of invasive species. Invasive Species Specialist Group of the World Conservation Union (IUCN), Auckland, New Zealand

Wooton JT (1992) Indirect effects, prey susceptibility, and habitat selection: impacts of birds on limpets and algae. Ecology 73:981-991

Zamorano JH (1986) Rattus rattus (Rodentia, Muridae) Un depredador intermareal poco conocido. Medio Ambient 8:58-62

Zieman JC, Thayer GW, Robblee MB, Zieman RT (1979) Production and export of sea grasses from a tropical bay. In: Livingston RJ (ed) Ecological processes in coastal and marine ecosystems. Plenum, New York, p 21-33

Submitted: January 13, 2003; Accepted: April 3, 2003

Proofs received from author(s): June 18, 2003 